\title{
Kinetic Electron-Ion Two Streams Instability in Space Dusty Plasma with Temperature Gradient
}

\author{
Sherif Mohamed Khalil ${ }^{1,2 *}$, Weeam Saleh Albaltan ${ }^{1}$ \\ ${ }^{1}$ Physics Department, Faculty of Science, Princess Nora Bent Abdurrahman University, Riyadh, Saudi Arabia \\ ${ }^{2}$ On Leave from Plasma Physics \& Nuclear Fusion Department, N.R.C., Atomic Energy Authority, Cairo, Egypt \\ Email: "smkhalil@pnu.edu.sa
}

Received 2 June 2015; accepted 30 November 2015; published 3 December 2015

Copyright (C) 2015 by authors and Scientific Research Publishing Inc.

This work is licensed under the Creative Commons Attribution International License (CC BY). http://creativecommons.org/licenses/by/4.0/

c) (i) Open Access

\section{Abstract}

The excitation, growing and damping of current instability is an important and vital subject for a lot of studies through its importance in communication for instance and in understanding the nature of space and the interpretation of many phenomena in space and astrophysics. Recent analytical and numerical works are presented to describe and investigate the excitation and growing of kinetic electron-ion two streams instability in anisotropic inhomogeneous dusty space plasmas. We elucidated the thermal effects of plasma species on the characteristics of such instability. It is found that the gradient of space plasma temperature, $\omega_{T \alpha}=\frac{k_{\perp} V_{T \alpha}^{2}}{\omega_{c \alpha}} \frac{\partial}{\partial x} \ln T_{\alpha}$, is a cause of interesting physical phenomena. Besides, different parameters, such as electron to ion temperature ratio $\frac{T_{e}}{T_{i}}$, magnetized plasma and dust grains, are also found to play a crucial role in the growth and depression of such instability.

\section{Keywords}

Electron-Ion Two Streams Instability, Anisotropic Space Plasma, Dusty Space Plasma

\section{Introduction}

The Buneman instability [1]-[7] is one of the current-driven plasma instabilities. The Buneman instability

\footnotetext{
${ }^{*}$ Corresponding author.
}

How to cite this paper: Khalil, S.M. and Albaltan, W.S. (2015) Kinetic Electron-Ion Two Streams Instability in Space Dusty Plasma with Temperature Gradient. International Journal of Astronomy and Astrophysics, 5, 248-257. 
represents the stimulated Cherenkov radiation of the low-frequency. The Buneman (electron-ion two streams) instability takes place in a current-driven system where electron and ion beams drift at different velocities. Strong electron streaming leads to the growing of Buneman instability. This growing mode leads to strong localized electric fields. The resulting electron scattering produces strong enhanced resistivity and electron heating [8].

From the experimental point of view, a rapidly growing of the ultra-high-frequency (UHF) (>250 MHz) wave burst, identified as the Buneman two-stream instability, was detected at the beginning of a high-current plasma discharge [9]. The Buneman instability operates in both magnetized and unmagnetized plasmas near the electron plasma frequency (in the electron reference frame) and is excited when the electron drift velocity exceeds the electron thermal velocity [10]-[12].

For plasma turbulent heating, plasma heating in discharges and closed magnetic traps, acceleration of plasmoids, and for producing self-induced electric fields in plasmas, strong electric fields are used. This causes currents $u=u_{e}-u_{i}$ due to the relative motion of electrons and ions. If $u$ is comparable to the electron thermal velocity $V_{T e}\left(V_{T e}=\sqrt{T_{e} / m_{e}}\right)$, the current Buneman's instability arises.

This type of instability is excited kinetically [13] [14], however, it is excited hydrodynamically when $u$ considerably exceeds a certain threshold value $\left(u>u_{c r} \approx V_{T e}\right)$. In this case, the development of the instability involves a displacement of the plasma regions and results in the variation of the spatial configuration of the plasma. On the other hand, near the threshold when $\Delta u=u-u_{c r}$, or when waves receive energy-due to Cherenkov resonance-from a small group of resonance particles, this instability becomes kinetic. In this case, the phase velocities of the waves are less by an order of magnitude than the thermal velocities of the particles.

As application, excluding the sheath regions, experiments in the magneto-plasmadynamic MPD thruster have shown that the electron drift velocity is only a small fraction of the electron thermal velocity, effectively stabilizing the Buneman instability. Streaming between reflected protons and upstream electrons gives rise to a strong Buneman instability [15] [16]. It is likely to attribute the Buneman instability and the modified two stream instability to play important roles in the production of high energy electrons at SNRs (supernova remnants). In astrophysical plasmas, the magnetic-reconnection Buneman instability may be excited [17].

Generally speaking, waves and instabilities occupy a significant part of modern plasma physics research because most properties of plasmas are related to the fundamental wave modes in laboratory and space plasma systems. In recent years, numerous studies showed interest in investigating dusty plasmas having electrons, ions, and charged dust grains of spherical shapes [18]-[23].

When dust grains are immersed in plasma, they become negatively charged because impinging electrons move faster than impinging ions. The negatively-charged dust grains can be considered as a third plasma species, so the plasma consists of electrons (negative), ions (positive), and dust grains (negative) [24].

In the presence of particle streaming, Jeans instability and the Buneman instability can overlap [25]. Further studies examined the influence of dust size distribution on the Jeans-Buneman instability [26]. Besides, the Buneman-type streaming instability may be developed in a low-temperature collisionless plasma in the presence of highly-charged impurities or dust and an ion flow. The important feature of the instability is that it takes place for supersonic as well as for subsonic velocities of the flow, thus being able to develop even in the pre-sheath and plasma bulk regions of low-temperature discharges where ion speeds are below the sound velocity [27].

Since charged dust in laboratory plasmas are generally levitated by electric fields, ions which acquire drifts due to these fields can stream through the dust, leading to various kinds of streaming instabilities, among them the Buneman instability. Recently, an ion-dust streaming instability with frequency less than the dust-neutral collision frequency was investigated [28]. The instability may have application to observations of waves in certain laboratory dc glow discharge dusty plasmas. Besides, dusty plasmas in the laboratory generally have finite spatial extent; therefore boundary effects may alter the properties of such ion-dust streaming instabilities. Recently, ion-dust streaming instability in plasma containing dust grains with large thermal speeds was considered using kinetic theory [29].

Buneman-type instabilities in a dusty plasma have been investigated before by many authors, e.g., ion-dust streaming instability in processing plasmas including collision effects [30], Buneman-type streaming instability in a plasma with dust particulates without collisions [27], and ion-dust two-stream instability in a collision including a magnetic field and collisions [31].

We extend previous work [13] [14] [32]-[37] for space plasma, with the following new consideration:

1) different temperature regimes (a mechanism for instability growth control), 
2) plasma temperature inhomogeneity,

3) space dusty plasmas.

\section{Space Plasma Kinetic Dispersion Relation}

Let us consider an inhomogeneous space plasma immersed in a static magnetic field $\boldsymbol{H}_{0}=\boldsymbol{e}_{Z} H_{0}$ with cold weakly magnetized dusts. Plasma temperature inhomogeneity is perpendicular to $H_{0}$, i.e., directed along the $\mathrm{x}$-axis.

In a dusty plasma, the quasi-neutrality condition should be adopted to include dust grains as:

$$
\sum_{\alpha}^{e, i, d} e_{\alpha} n_{\alpha 0}=0
$$

For low $\beta$ (the ratio of thermal to magnetic pressure), the kinetic dispersion relation governing the system is:

$$
\begin{gathered}
\varepsilon(\boldsymbol{k}, \omega)=1+\sum_{\alpha}^{e, i, d} \chi_{\alpha}=0 \\
\chi_{\alpha}=\frac{\omega_{p \alpha}^{2}}{k_{\|}^{2} V_{T \alpha}^{2}}\left[1+i \sqrt{\pi} \hat{l}_{\alpha} Z_{\alpha} W\left(Z_{\alpha}\right) A\left(\mu_{\alpha}\right)\right]
\end{gathered}
$$

$k_{\|}$is the wave number parallel to $\boldsymbol{H}_{0}, \chi_{\alpha}$ are the plasma susceptibilities, $\alpha$ is the type of particles, i.e., $\alpha=e, i, d$, for electrons, ions and dusts, respectively. The operator $\hat{l}_{\alpha}$ represents the effect of plasma inhomogeneity in density and temperature,

$$
\hat{l}_{\alpha}=1-\frac{k_{\perp} V_{T \alpha}^{2}}{\left(\omega-\boldsymbol{k} \boldsymbol{u}_{\alpha}\right) \omega_{c \alpha}}\left[\frac{\partial}{\partial x} \ln n_{\alpha}+\frac{\partial}{\partial x} \ln T_{\alpha}\right]
$$

Here, we are interested in the temperature inhomogeneity, i.e., $\partial \ln n_{\alpha} / \partial x=0 . A\left(\mu_{\alpha}\right)$ represents the magnetic field effect, $A\left(\mu_{\alpha}\right)=I_{0}\left(\mu_{\alpha}\right) e^{-\mu_{\alpha}}, \mu_{\alpha}=k_{\perp}^{2} \rho_{\alpha}^{2}, k_{\perp}$ the wave number perpendicular to $\boldsymbol{H}_{0}$, $\rho_{\alpha}=V_{T \alpha} / \omega_{c \alpha}$ is the Larmor radius, $\omega_{c \alpha}=e_{\alpha} H_{0} /\left(m_{\alpha} c\right)$ is the cyclotron frequency, $V_{T \alpha}=\sqrt{T_{\alpha} / m_{\alpha}}$ the thermal velocity, $I_{0}\left(\mu_{\alpha}\right)$ is the modified Bessel function of the $0^{\text {th }}$ order, and $\omega_{p \alpha}=\sqrt{4 \pi e^{2} n_{\alpha}(x) / m_{\alpha}}$ is the Langmuir frequency. $W\left(Z_{\alpha}\right)$ is the known probability integral with amplitude:

$$
Z_{\alpha}=\frac{\omega-\boldsymbol{k} \cdot \boldsymbol{u}_{\alpha}}{\sqrt{2} k_{\|} V_{T \alpha}}
$$

If $\boldsymbol{k} \cdot \boldsymbol{u}_{i} \approx \boldsymbol{k} \cdot \boldsymbol{u}_{d}\left(\boldsymbol{u}_{i} \approx \boldsymbol{u}_{d}\right)$, and we set $\grave{\omega}=\omega-\boldsymbol{k} \cdot \boldsymbol{u}_{d}=\omega-\boldsymbol{k} \cdot \boldsymbol{u}_{i}$, then $Z_{\alpha}$ for the different plasma different species are:

$$
Z_{e}=\frac{\grave{\omega}-\boldsymbol{k} \cdot \boldsymbol{u}}{\sqrt{2} k_{\|} V_{T e}}, Z_{(i, d)}=\frac{\grave{\omega}}{\sqrt{2} k_{\|} V_{T(i, d)}}
$$

where $\boldsymbol{k} \cdot \boldsymbol{u}=k u \cos \theta$, and $\theta$ is the angle between $\boldsymbol{k}$ and $\boldsymbol{u}=\boldsymbol{u}_{e}-\boldsymbol{u}_{i}$. We consider dusty plasma consisting of fairly massive grains, i.e., $m_{d} / m_{i} \gg 1$.

\section{Threshold of Instability}

For the excitation of instability we set

$$
\begin{gathered}
\grave{\omega}=\omega_{0}+\Delta \omega,|\Delta \omega| \ll \omega_{0}, \Delta \omega=\omega_{k}+i \gamma_{k} \\
u=u_{c r}+\Delta u, \cos \theta=1-\frac{\theta^{2}}{2},|\theta| \ll 1
\end{gathered}
$$

The values $\Delta \omega, \Delta u, \theta^{2}$ are small perturbations, used for the expansion of 


$$
Z_{\alpha}=Z_{0 \alpha}+\Delta Z_{\alpha},\left|\Delta Z_{\alpha}\right| \ll Z_{0 \alpha} ; \alpha=e, i, d
$$

where $\omega_{k}$ and $\gamma_{k}$ are the frequency and growth rate of the instability, respectively, and $u_{c r}$ and $\omega_{0}$ are the threshold values of current velocity and frequency. For such perturbations, we have $\frac{\Delta u}{u_{c r}}, \frac{\Delta \omega}{\omega}, \frac{\Delta k}{k}, \theta \ll 1$.

Now, for weakly magnetized dusts, $\mu_{d} \ll 1$, and under the frequency ranges $\left|\boldsymbol{k} \cdot \mathbf{u}_{i}, \boldsymbol{k} \cdot \boldsymbol{u}_{d}\right| \ll \grave{\omega},|\boldsymbol{k} \cdot \boldsymbol{u}| \ll \grave{\omega}$, and validity of the parameters

$$
n_{d} \ll n_{e} \approx n_{i}, \frac{n_{d}}{n_{e, i}} \leq 1 ; Z_{0 i} \approx Z_{0 d} \gg Z_{0 e} ; Z_{0 i} \gg 1, Z_{0 e} \ll 1,
$$

we get

$$
\begin{gathered}
A\left(\mu_{i}\right) \cong \frac{1}{\sqrt{2 \pi \mu_{i}}}, \quad A\left(\mu_{e}\right) \cong 1-\mu_{e}, A\left(\mu_{d}\right) \cong 1 \\
\hat{l}_{e} \approx 1-\frac{\omega_{T e}}{\grave{\omega}}, \hat{l}_{i} \approx 1-\frac{\omega_{T i}}{\grave{ }}, \hat{l}_{d} \approx 1-\frac{\omega_{T d}}{\grave{\omega}}
\end{gathered}
$$

where

$$
\begin{gathered}
\omega_{T \alpha}=\frac{k_{\perp} V_{T \alpha}^{2} \kappa_{T \alpha}}{\omega_{c \alpha}}, \kappa_{T \alpha}=\frac{\partial}{\partial x} \ln T_{\alpha} ; \\
V_{T \alpha}^{2}=\frac{T_{\alpha}}{m_{\alpha}}
\end{gathered}
$$

Regardless the temperature ratio $T_{d} / T_{i}$, for a dusty plasma, we have:

$$
\frac{\rho_{d}}{\rho_{i}}=\sqrt{\frac{T_{d}}{T_{i}}} \sqrt{\frac{m_{d}}{m_{i}}} \gg 1
$$

In linear regime, and under the specific conditions mentioned above (which agrees with experiment), the dispersion relation (1) reads:

$$
\begin{aligned}
& 1+\frac{T_{e}}{T_{i}}+k^{2} r_{d e}^{2}+i \sqrt{\pi}\left(1-\frac{\omega_{T e}}{\grave{\omega}}\right) Z_{e} W\left(Z_{e}\right)\left(1-\mu_{e}\right) \\
& +\frac{T_{e}}{T_{i}} i \sqrt{\pi}\left(1-\frac{\omega_{T i}}{\grave{\omega}}\right) Z_{i} W\left(Z_{i}\right) \frac{1}{\sqrt{2 \pi \mu_{i}}} \\
& +\frac{T_{e}}{T_{d}} \frac{n_{d}}{n_{e}}\left[1+i \sqrt{\pi}\left(1-\frac{\omega_{T d}}{\omega}\right) Z_{d} W\left(Z_{d}\right)\right]=0
\end{aligned}
$$

where $r_{\mathrm{de}}=V_{T e} / \omega_{\text {pe }}$ is the electron Debye radius.

Assuming in (2), $\omega^{\prime} \rightarrow \omega_{0}$ and $u \rightarrow u_{c r}$, we obtain the threshold velocity of instability as:

$$
\begin{aligned}
u_{c r}= & \sqrt{\frac{\pi}{8}} \frac{V_{T_{i}} \omega_{0}}{\omega_{c i}}\left(1-\mu_{e}\right) e^{z_{0 i}^{2}}\left(\frac{V_{T_{i}}}{V_{T_{e}}}\right)^{3} \frac{m_{i}}{m_{e}}\left(1-\frac{\omega_{T_{e}}}{\omega_{0}}\right)\left(1-\frac{\omega_{T_{i}}}{\omega_{0}}\right)^{-1} \\
& \cdot\left[1+\left(\frac{V_{T_{e}}}{V_{T_{d}}}\right)^{3} \frac{m_{e}}{m_{d}} \frac{n_{d}}{n_{e}} \frac{1}{\left(1-\mu_{e}\right)} e^{-z_{0 d}^{2}}\left(1-\frac{\omega_{T_{d}}}{\omega_{0}}\right)\left(1-\frac{\omega_{T_{e}}}{\omega_{0}}\right)^{-1}\right]^{2}
\end{aligned}
$$

It is clear that this instability depend strongly on the following parameters: plasma dust, temperature inhomogeneity $\left(\omega_{T_{e}}, \omega_{T_{i}}\right.$ and $\omega_{T_{d}}$ ). Figure 1 shows clearly these different dependencies. 

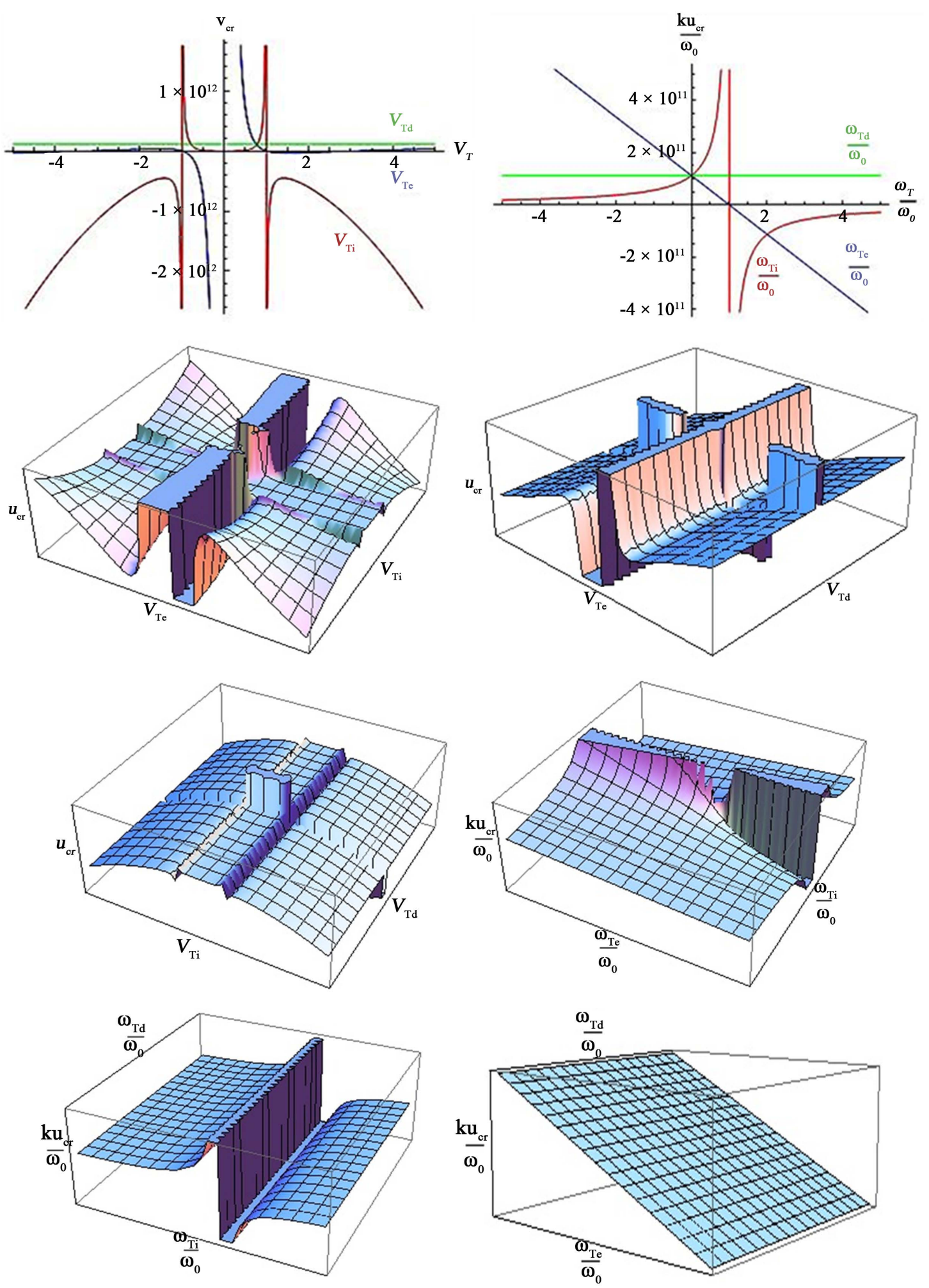

Figure 1. Critical velocity against ion and electron thermal velocities, and dusty particles; Critical current velocity against electrons and ions thermal velocities; Critical current velocity against electrons and dust thermal velocities; Critical current velocity against ions and dust thermal velocities, Critical current frequency against electrons and ions thermal frequencies due to inhomogeneity; Critical current frequency against ions and dust thermal frequencies due to inhomogeneity; Critical current frequency against electrons and dust thermal frequencies due to inhomogeneity. 


\section{Special cases}

Table 1 declares the effect of temperature inhomogeneity on the threshold current velocity of the instability for different cases:

where

$$
\begin{gathered}
\aleph=\sqrt{\frac{\pi}{8}} \frac{V_{T_{i}} \omega_{0}}{\omega_{c i}}\left(1-\mu_{e}\right) e^{z_{0 i}^{2}}\left(\frac{V_{T_{i}}}{V_{T_{e}}}\right)^{3} \frac{m_{i}}{m_{e}}\left(1-\frac{\omega_{T_{i}}}{\omega_{0}}\right)^{-1} \\
\mathcal{M}=\left(\frac{V_{T_{e}}}{V_{T_{d}}}\right)^{3} \frac{m_{e}}{m_{d}} \frac{n_{d}}{n_{e}} \frac{1}{\left(1-\mu_{e}\right)} e^{-z_{0 d}^{2}}\left(1-\frac{\omega_{T_{d}}}{\omega_{0}}\right)
\end{gathered}
$$

The table shows clearly the temperature inhomogeneity controlling, accordingly the initiation of current instability. For example, strong inhomogeneity in the dust temperature increases the $u_{c r}$, hence delaying appearance of instability.

\section{Excitation of Instability}

The excitation of Buneman instability put the plasma into a strongly turbulent state. Then a strong turbulent heating of the plasma leads to a rapid increase of the threshold current $u_{c r}$ of the instability up to a value $u$, Therefore, it will be of great interest to investigate here this instability at current velocities close to the threshold values.

Accordingly, let us consider that the current velocity slightly exceeds the instability threshold, i.e.,

$$
u=u_{c r}+\Delta u \text {, }
$$

where $\frac{\Delta u}{u_{c r}} \ll 1$.

Now, for the excitation of instability $\gamma_{k}, \omega_{k} \neq 0$. Accordingly, the dispersion (1) takes the general form:

Table 1. Effect of temperature inhomogeneity on the threshold current velocity of the instability.

$$
\text { Case } \quad\left|u_{c r}\right|=
$$

1

$$
\frac{\omega_{T_{e}}}{\omega_{0}} \ll 1
$$$$
\aleph(1+\mathcal{M})^{2}
$$

2

$$
\frac{\omega_{T_{e}}}{\omega_{0}} \gg 1
$$

$$
\aleph\left(\frac{\omega_{T_{e}}}{\omega_{0}}\right)\left(1+\left(\frac{\omega_{0}}{\omega_{T_{e}}}\right) \mathcal{M}\right)^{2}
$$

3

$$
\frac{\omega_{T_{i}}}{\omega_{0}} \ll 1
$$

$$
\aleph\left(1-\frac{\omega_{T_{i}}}{\omega_{0}}\right)^{2}\left(1+\left(1-\frac{\omega_{T_{e}}}{\omega_{0}}\right)^{-1} \mathcal{M}\right)^{2}
$$

4

$$
\frac{\omega_{T_{i}}}{\omega_{0}} \gg 1
$$

$$
\aleph\left(1-\frac{\omega_{T_{i}}}{\omega_{0}}\right)^{2}\left(\frac{\omega_{0}}{\omega_{T_{i}}}\right)\left(1+\left(1-\frac{\omega_{T_{e}}}{\omega_{0}}\right)^{-1} \mathcal{M}\right)^{2}
$$

$5 \quad \frac{\omega_{T_{d}}}{\omega_{0}} \ll 1$

$$
\aleph\left(1-\frac{\omega_{T_{e}}}{\omega_{0}}\right)\left(1+\left(1-\frac{\omega_{T_{e}}}{\omega_{0}}\right)^{-1}\left(1-\frac{\omega_{T_{d}}}{\omega_{0}}\right)^{-1} \mathcal{M}\right)^{2}
$$

$6 \quad \frac{\omega_{T_{d}}}{\omega_{0}} \gg 1$

$$
\aleph\left(1-\frac{\omega_{T_{e}}}{\omega_{0}}\right)\left(1-\left(1-\frac{\omega_{T_{e}}}{\omega_{0}}\right)^{-1}\left(1-\frac{\omega_{T_{d}}}{\omega_{0}}\right)^{-1} \mathcal{M}\right)^{2}
$$




$$
\begin{aligned}
& \sqrt{\pi}\left(1-\mu_{e}\right)\left(1-\frac{\omega_{T e}}{\omega_{0}}\right) \mathcal{R}_{0 e} \frac{\omega^{\prime}+\mathcal{W}}{\sqrt{2} k_{\|} v_{T_{e}}}+\frac{T_{e}}{T_{i}} \frac{1}{\sqrt{2 \mu_{i}}}\left(1-\frac{\omega_{T i}}{\omega_{0}}\right) \mathcal{R}_{0 i} \frac{\omega^{\prime}}{\sqrt{2} k_{\|} v_{T_{i}}} \\
& +\frac{T_{e}}{T_{d}} \frac{n_{d}}{n_{e}} \sqrt{\pi}\left(1-\frac{\omega_{T d}}{\omega_{0}}\right) \mathcal{R}_{0 d} \frac{\omega^{\prime}}{\sqrt{2} k_{\|} v_{T_{d}}}+i \sqrt{\pi}\left(1-\mu_{e}\right) \frac{\omega_{T e} \omega^{\prime}}{\omega_{0}^{2}} Z_{0 e} e^{-Z_{0 e}^{2}} \\
& -\left(1-\mu_{e}\right) \frac{\omega_{T e} \omega^{\prime}}{\omega_{0}^{2}}+\frac{T_{e}}{T_{i}} i \frac{1}{\sqrt{2 \mu_{i}}} \frac{\omega_{T i} \omega^{\prime}}{\omega_{0}^{2}} Z_{0 i} e^{-Z_{0 i}^{2}}-\frac{T_{e}}{T_{i}} \frac{1}{\sqrt{2 \pi \mu_{i}}} \frac{\omega_{T i} \omega^{\prime}}{\omega_{0}^{2}}+\frac{T_{e}}{T_{d}} \frac{n_{d}}{n_{e}} \frac{\omega_{T d} \omega^{\prime}}{\omega_{0}^{2}}\left[i \sqrt{\pi} Z_{0 d} e^{-Z_{0 d}^{2}}-1\right]=0
\end{aligned}
$$

where $\mathcal{W}=\left[\frac{\Delta u}{u_{c r}}-\frac{\theta^{2}}{2}\right] ; \quad \omega^{\prime}=\omega_{k}+i \gamma_{k}$ and $\mathcal{R}_{0 \alpha}=i\left(1-2 Z_{0 \alpha}^{2}\right) e^{-Z_{0 \alpha}^{2}}-\frac{1}{\sqrt{\pi} Z_{0 \alpha}}$

The dispersion relation (4) yields the growth rate and the frequency (high frequency) of Buneman instability in inhomogeneous dusty space plasma for different temperature regimes as follows:

$$
\begin{aligned}
& \gamma_{k}=\left\{\mathbb{O}_{1}-\sqrt{\pi} Z_{0 e} \mathbb{O}_{2}+\frac{n_{d}}{n_{e}}\left[\mathbb{N}_{1}+\mathbb{N}_{2}\right]\right\} \mathbb{R} \\
& \omega_{k}=\left\{\mathbb{O}_{2}+\sqrt{\pi} Z_{0 e} \mathbb{O}_{1}+\frac{n_{d}}{n_{e}}\left[\mathbb{N}_{1}+\mathbb{N}_{2}\right]\right\} \mathbb{R}
\end{aligned}
$$

where,

$$
\begin{gathered}
\mathbb{R}=\left\{\frac{\frac{T_{i}}{T_{e}}\left(1-\mu_{e}\right) \sqrt{2 \mu_{i}}\left(\frac{\omega_{T e}}{\omega_{0}}-1\right) \frac{1}{Z_{0 e}} \omega_{0} \frac{k u_{c r} \mathcal{W}}{\sqrt{2} k_{\|} v_{T_{e}}}}{\mathbb{O}_{1}^{2}+\mathbb{O}_{2}^{2}+\frac{n_{d}}{n_{e}}\left[\frac{n_{d}}{n_{e}}\left(\mathbb{N}_{1}^{2}+\mathbb{N}_{2}^{2}\right)+2\left(\mathbb{O}_{1} \mathbb{N}_{1}+\mathbb{O}_{2} \mathbb{N}_{2}\right)\right]}\right\} \\
\mathbb{O}_{1}=Z_{0 i} e^{-Z_{0 i}^{2}}\left[2 Z_{0 i}^{2}\left(1-\frac{\omega_{T i}}{\omega_{0}}\right)+\frac{\omega_{T e}}{\omega_{0}}\left(1-\frac{\omega_{T i}}{\omega_{0}}\right)\left(1-\frac{\omega_{T_{e}}}{\omega_{0}}\right)^{-1}\right] \\
\mathbb{N}_{1}=\frac{T_{i}}{T_{d}} \sqrt{2 \pi \mu_{i}} Z_{0 d} e^{-Z_{0 d}^{2}}\left\{\left(1-\frac{1}{\sqrt{\pi}}\left[1-\left(1-\frac{\omega_{T d}}{\omega_{0}}\right)\left(1-\frac{\omega_{T_{e}}}{\omega_{0}}\right)^{-1} \frac{\omega_{T e}}{\omega_{0}} \frac{Z_{0 i}}{Z_{0 e}} e^{-Z_{0 i}^{2}}\right]\right.\right. \\
\left.\left.\mathbb{N}_{2}=\frac{\omega_{T_{e}}}{\omega_{0}}\left(1-\frac{\omega_{T_{e}}}{\omega_{0}}\right)^{-1}+2 Z_{0 i}^{2}\right]-\frac{\omega_{T d}}{\omega_{0}}\right\} \\
\sqrt{2 \mu_{i}}\left\{\left(1-\frac{\omega_{T d}}{\omega_{0}}\right)\left[\frac{\omega_{T_{e}}}{\omega_{0}} \frac{Z_{0 d}}{Z_{0 e}} e^{-Z_{0 d}^{2}}\left(1-\frac{\omega_{T_{e}}}{\omega_{0}}\right)^{-1}-1\right]-\frac{\omega_{T d}}{\omega_{0}}\right\}
\end{gathered}
$$

The growth rate $\gamma_{k}$ against the temperature gradients $\omega_{T d}, \omega_{T e}$ and $\omega_{T i}$ are shown clearly in Figure 2.

The growth rate and frequency of the instability for special cases of inhomogeneous magnetized dusty space plasma are investigated, i.e., for:

1) Weak and strong electrons temperature inhomogeneity $\left(\frac{\omega_{T_{e}}}{\omega_{0}} \ll 1 ; \frac{\omega_{T_{e}}}{\omega_{0}} \gg 1\right)$

2) Weak and strong ions temperature inhomogeneity $\left(\frac{\omega_{T_{i}}}{\omega_{0}} \ll 1 ; \frac{\omega_{T_{i}}}{\omega_{0}} \gg 1\right)$

3) Weak and strong ions temperature inhomogeneity $\left(\frac{\omega_{T_{d}}}{\omega_{0}} \ll 1 ; \frac{\omega_{T_{d}}}{\omega_{0}} \gg 1\right)$

We obtained the following relations: 

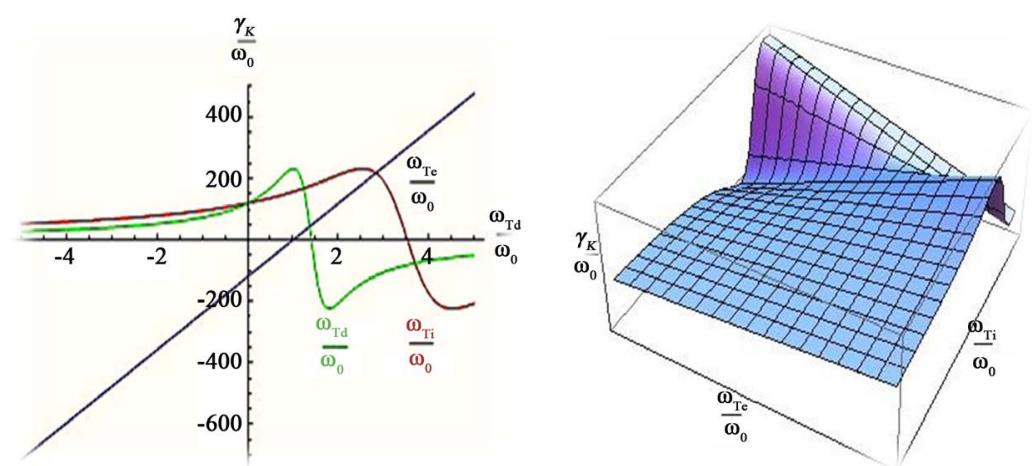

Figure 2. Growth rate $\gamma_{k}$ against temperature inhomogeneity $\omega_{T d}, \omega_{T e}$ and $\omega_{T i}$.

$$
\frac{\gamma_{k}\left(\frac{\omega_{T e}}{\omega_{0}} \ll 1\right)}{\gamma_{k}\left(\frac{\omega_{T e}}{\omega_{0}} \gg 1\right)}<1, \frac{\gamma_{k}\left(\frac{\omega_{T i}}{\omega_{0}} \ll 1\right)}{\gamma_{k}\left(\frac{\omega_{T i}}{\omega_{0}} \gg 1\right)}>1 \text { and } \frac{\gamma_{k}\left(\frac{\omega_{T d}}{\omega_{0}} \ll 1\right)}{\gamma_{k}\left(\frac{\omega_{T d}}{\omega_{0}} \gg 1\right)}>1
$$

\section{Discussion and Conclusions}

In this paper, analytical and numerical works are presented to describe and investigate the excitation and growing of kinetic electron-ion two streams instability in the anisotropic inhomogeneous dusty space plasmas. We elucidated the thermal effects of plasma species on the characteristics of such instability.

It is found that the gradient of space plasma temperature, $\omega_{T \alpha}=\frac{k_{\perp} V_{T \alpha}^{2}}{\omega_{c \alpha}} \frac{\partial}{\partial x} \ln T_{\alpha}$, is a cause of interesting physical phenomena. Besides, different parameters, such as electron to ion temperature ratio $\frac{T_{e}}{T_{i}}$, magnetized plasma and dust grains, are found to play a crucial role in the growth and depression of such instability.

Thermal velocities of electrons and ions affect strongly the lagging and leading of instability according to the relations, $\left(\frac{V_{T i}}{u_{c r}} \ll 1\right) ;\left(\frac{V_{T e}}{u_{c r}} \gg 1\right)$, respectively.

This result is also confirmed by the following relations

$$
\frac{u_{c r}\left(\frac{\omega_{T e}}{\omega_{0}} \ll 1\right)}{u_{c r}\left(\frac{\omega_{T e}}{\omega_{0}} \gg 1\right)}>1 ; \frac{u_{c r}\left(\frac{\omega_{T i}}{\omega_{0}} \ll 1\right)}{u_{c r}\left(\frac{\omega_{T i}}{\omega_{0}} \gg 1\right)}>1
$$

which show that the frequencies due to temperature inhomogeneity $\omega_{T e}, \omega_{T i}$ influence the critical velocity $u_{c r}$ of the instability.

$\omega_{T e}, \omega_{T i}$ and $\omega_{T d}$, are found to play a crucial role in the growth rate of the instability (increasing or decreasing) according to the relations:

$$
\frac{\gamma_{k}\left(\frac{\omega_{T e}}{\omega_{0}} \ll 1\right)}{\gamma_{k}\left(\frac{\omega_{T e}}{\omega_{0}} \gg 1\right)}<1 ; \frac{\gamma_{k}\left(\frac{\omega_{T i}}{\omega_{0}} \ll 1\right)}{\gamma_{k}\left(\frac{\omega_{T i}}{\omega_{0}} \gg 1\right)}>1 ; \frac{\gamma_{k}\left(\frac{\omega_{T d}}{\omega_{0}} \ll 1\right)}{\gamma_{k}\left(\frac{\omega_{T d}}{\omega_{0}} \gg 1\right)}>1
$$

This shows that temperature inhomogeneity is a mechanism for growing or damping of the instability.

Another mechanism is the magnetic field. As in Figure 3 the relations between the cyclotron frequencies $\omega_{c e}$, 


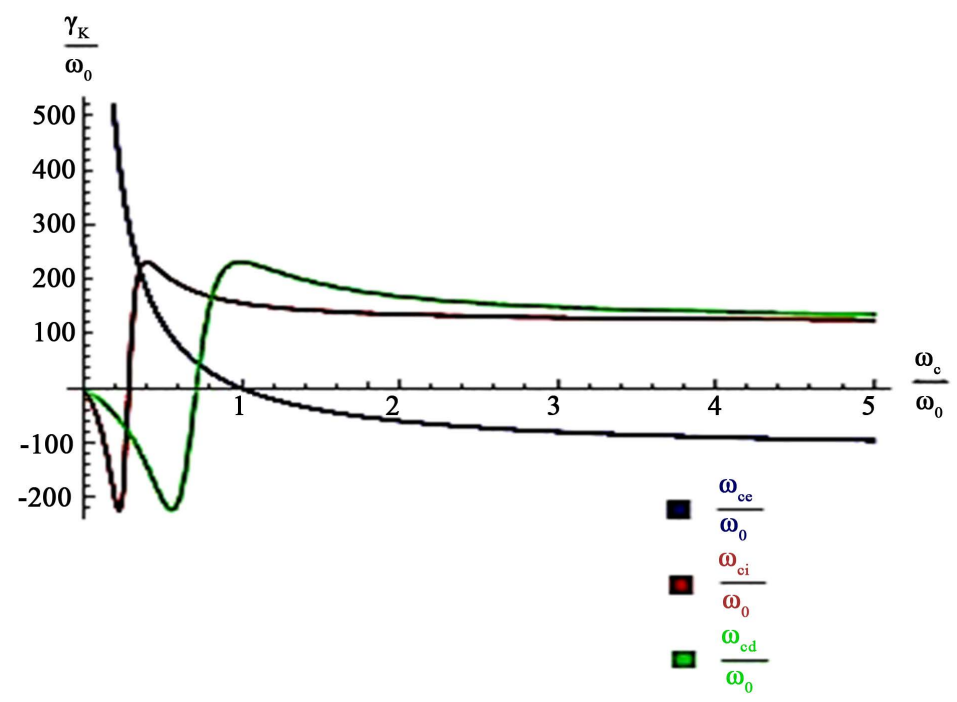

Figure 3. Growth rates $\gamma_{k}$ against cyclotron frequencies $\omega_{c e}, \omega_{c i}, \omega_{c d}$.

$\omega_{c i}, \omega_{c d}$, and growth rate $\gamma_{k}$ are shown.

It is clear that magnetized electron is a good mechanism for depression and saturation of such instability. This mechanism is also applicable for extremely strong magnetized ions and dust.

Under investigation is the nonlinear and multi-ion species effect on the dispersion characteristics of such instability.

\section{References}

[1] Buneman, O. (1958) Instability, Turbulence, and Conductivity in Current-Carrying Plasma. Physics Review Letters, 1, 8. http://dx.doi.org/10.1103/PhysRevLett.1.8

[2] Alexandrov, A.F., Bogdankevich, L.S. and Rukhadze, A.A. (1984) Principles of Plasma Electrodynamic. SpringerVerlag, Berlin. http://dx.doi.org/10.1007/978-3-642-69247-5

[3] Raizer, Y.P. (1991) Gas Discharge Physics. Springer-Verlag, New York. http://dx.doi.org/10.1007/978-3-642-61247-3

[4] Iizuka, S., Saeki, K., Sato, N. and Hatta, Y.J. (1985) Buneman Instability in a Bounded Electron Beam-Plasma System. Journal of the Physical Society of Japan, 54, 146. http://dx.doi.org/10.1143/JPSJ.54.146

[5] Shokri, B. and Vazifehshenas, T. (2001) Thermal Motion Effect on the Filamentation of a Strongly Collisional Current-Driven Plasma. Physics of Plasmas, 8, 788. http://dx.doi.org/10.1063/1.1339231

[6] Shokri, B., Khorashadi, S.M. and Dastmalchi, M. (2002) Ion-Acoustic Filamentation of a Current-Driven Plasma. Physics of Plasmas, 9, 3355. http://dx.doi.org/10.1063/1.1490133

[7] Shokri, B. and Niknam, A.R. (2003) Discharge Plasma Instabilities in the Presence of an External Constant Electric Field. Physics of Plasmas, 10, 4153. http://dx.doi.org/10.1063/1.1609990

[8] Drake, J. F., Shay, M.E., et al. (2001) Kinetic Treatment of Collisionless Reconnection. Bull Am. Phys. Soc., 46, 144.

[9] Takeda, Y., Inuzuka, H. and Yamagiwa, K. (1995) Observations of Buneman Modes as Precursory Phenomena of a Solitary Potential Pulse. Physics Review Letters, 74, 1998. http://dx.doi.org/10.1103/PhysRevLett.74.1998

[10] Lemons, D.S. and Gary, S.P. (1978) Current-Driven Instabilities in a Laminar Perpendicular Shock. Journal of Geophysical Research, 831, 1625. http://dx.doi.org/10.1029/JA083iA04p01625

[11] Haihong Che, Goldman, M.V. and Newman, D.L. (2011) Buneman Instability in a Magnetized Current-Carrying Plasma with Velocity Shear. Physics of Plasmas, 18, 5. http://dx.doi.org/10.1063/1.3590879

[12] Bohata, M., Břeň, D. and Kulhánek, P. (2011) Generalized Buneman Dispersion Relation in the Longitudinally Dominated Magnetic Field. ISRN Condensed Matter Physics, 2011, Article ID: 896321.

[13] Hussein, A.M., Khalil, S.M. and Sizonenko, V.L. (1978) On the Theory of Turbulence in Weak Magneto-Active Plasma. Plasma Physics, 20, 545-560. http://dx.doi.org/10.1088/0032-1028/20/6/005

[14] El-Naggar, I.A., Khalil, S.M. and Sizonenko, V.L. (1978) On the Theory of Buneman’s Instability in Hot Ion Plasma. Journal of Plasma Physics, 20, 75-85. http://dx.doi.org/10.1017/S0022377800021371 
[15] Drury, L. O’C., McClements, K.G., Chapman, S.C., Dendy, R.O., Dieckmann, M.E., Ljung, P. and Ynnerman, A. (2001) Computational Studies of Cosmic Ray Electron Injection. Proceedings of the 27th International Cosmic Ray Conference, Hamburg, 7-15 August 2001, 2096.

[16] Dieckmann, M.E., McClements, K.G., Chapman, S.C., et al. (2000) Electron Acceleration Due to High Frequency Instabilities at Supernova Remnant Shocks. Astronomy and Astrophysics, 356, 377-388.

[17] Sakai, J.I., Sugiyama, D., Haruki, T., et al. (2001) Magnetic Field Energy Dissipation Due to Particle Trapping in a Force-Free Configuration of Collisionless Pair Plasmas. Physical Review E, 63, Article ID: 046408. http://dx.doi.org/10.1103/PhysRevE.63.046408

[18] Salimullah, M., Shukla, P.K., Sandberg, I. and Morfill, G.E. (2003) Excitation of Dipole Oscillons in a Dusty Plasma Containing Elongated Dust Rods. New Journal of Physics, 5, 40. http://dx.doi.org/10.1088/1367-2630/5/1/340

[19] Shukla, P.K. and Mamun, A.A. (2002) Introduction to Dusty Plasma Physics. Institute of Physics Publishing, Bristol. http://dx.doi.org/10.1887/075030653X

[20] Mendis, D.A. (2002) Progress in the Study of Dusty Plasmas. Plasma Sources Science and Technology, 11, A219A228. http://dx.doi.org/10.1088/0963-0252/11/3A/333

[21] Scales, W.A. (2004) Nonlinear Development of a Low-Frequency Hall Current Instability in a Dusty Plasma. Physica Scripta, T107, 107. http://dx.doi.org/10.1238/Physica.Topical.107a00107

[22] D’Angelo, N. and Merlino, R.L. (1996) Current-Driven Dust-Acoustic Instability in a Collisional Plasma. Planetary and Space Science, 44, 1593-1598. http://dx.doi.org/10.1016/S0032-0633(96)00069-4

[23] Rosenberg, M. and Shukla, P.K. (1999) Instability of Ion Flows in Bounded Dusty Plasma Systems. Physics of Plasmas, 5, 3786. http://dx.doi.org/10.1063/1.872743

[24] Bellan, P.M. (2004) A Model for the Condensation of a Dusty Plasma. Physics of Plasmas, 11, 3368. http://dx.doi.org/10.1063/1.1740773

[25] Pandey, B.P. and Lakhina, G.S. (1998) Article title. Pramana-Journal of Physics, 50, 191.

[26] Meuris, P., Verheest, F. and Lakhina, G.S. (1997) Influence of Dust Mass Distributions on Generalized Jeans-Buneman Instabilities in Dusty Plasmas. Planetary and Space Science, 45, 449-454. http://dx.doi.org/10.1016/S0032-0633(96)00155-9

[27] Vladimirov, S.V. and Ishihara, O. (1999) Buneman-Type Streaming Instability in a Plasma with Dust Particulates. Physica Scripta, 60, 370-372. http://dx.doi.org/10.1238/Physica.Regular.060a00370

[28] Rosenberg, M. (2002) A Note on Ion-Dust Streaming Instability in a Collisional Dusty Plasma. Journal of Plasma Physics, 67, 235-242. http://dx.doi.org/10.1017/S0022377802001678

[29] Rosenberg, M., Thomas, E. and Merlino, R.L. (2008) A Note on Dust Wave Excitation in a Plasma with Warm Dust: Comparison with Experiment. Physics of Plasmas, 15, Article ID: 073701. http://dx.doi.org/10.1063/1.2943218

[30] Rosenberg, M. (1996) Ion-Dust Streaming Instability in Processing Plasmas. Journal of Vacuum Science \& Technology A, 14, 631. http://dx.doi.org/10.1116/1.580157

[31] Mamun, A. A. and Shukla, P. K. (2000) Streaming Instabilities in a Collisional Dusty Plasma. Physics of Plasmas, 7, 4412. http://dx.doi.org/10.1063/1.1315305

[32] Rosenberg, M. and Shukla, P.K. (2004) Ion-Dust Two-Stream Instability in a Collisional Magnetized Dusty Plasma. Journal of Plasma Physics, 70, 317-322. http://dx.doi.org/10.1017/S0022377803002678

[33] Khalil, S.M. and Mohamed, B.F. (1989) Quasilinear Theory of Buneman's Instability in Hot Electron Plasma $T_{e}>>T_{i}$. Proceedings of the 16th European Conference on Controlled Fusion and Plasma Physics, Venice, 13-17 March 1989, 1369.

[34] Khalil, S.M. and Mohamed, B.F. (1988) Electron-Ion Two-Stream Instability in Anisotropic Isothermal Plasma. Journal de Physique, 49, 451-455. http://dx.doi.org/10.1051/jphys:01988004903045100

[35] Khalil, S.M. and Mohamed, B.F. (1991) Kinetic Buneman Instability in a Homogeneous Hot Electron Plasma. Contributions to Plasma Physics, 31, 513-518. http://dx.doi.org/10.1002/ctpp.2150310506

[36] Khalil, S.M. and Sayed, Y.A. (1994) Kinetic Nonlinear Buneman's Instability in Field-Free Isothermal Collisonal Plasma. Contributions to Plasma Physics, 34, 683-689. http://dx.doi.org/10.1002/ctpp.2150340508

[37] Khalil, S.M. and Al-Yousef, H. (2010) Kinetic Buneman Instability in Dusty Plasma of Different Temperature Regimes. FIZIKA, A19, 1. 